\title{
A Tunable Diode-laser Absorption Spectroscopy (TDLAS) Thermometry for Combustion Diagnostics
}

\author{
X. L. Yu ${ }^{*}, \mathrm{~F} . \mathrm{Li}^{\dagger}$, L. H. Chen ${ }^{\ddagger}, \mathrm{X} . \mathrm{Y} . \mathrm{Chang}^{\S}$ \\ Institute of Mechanics, Chinese Academy of Sciences, Beijing100190, China
}

\begin{abstract}
A sensor based on tunable diode laser absorption spectroscopy (TDLAS) was constructed for non-intrusive temperature and water vapor concentration measurement. Absorption lines were selected and analysed using spectrum database Hitran2004. Two fiber-coupled near infrared distributed feedback (DFB) lasers was used to probe two water vapor absorption features $\left(7168.437 \mathrm{~cm}^{-1}\right.$ and $\left.7185.597 \mathrm{~cm}^{-1}\right)$ near $1393 \mathrm{~nm}$ which has high sensitivity of temperature measurement in $500-1300 \mathrm{~K}$. Successful experiments were performed in a $\mathrm{CH}_{4} /$ Air premixed flat flame burner using direct absorption wavelength scanned method at a $1 \mathrm{KHz}$ scan rate. Comparison with thermocouple measurements, the maxium error of the method is less than $80 \mathrm{~K}(6.7 \%)$.
\end{abstract}

\section{Nomenclature}

$I_{v, 0} \quad=$ incident light intensity

$I_{v} \quad=$ transmission light intensity

$k_{v} \quad=$ absorption coefficient, $\mathrm{cm}^{-1}$

$L=$ absorption length, $\mathrm{cm}$

$\phi(v) \quad=$ line shape function

$v \quad=$ light frequency, $\mathrm{cm}^{-1}$

$P_{a b s} \quad=$ pressure of absorbing species

$Q \quad=$ partition function

$E_{i}^{\prime \prime} \quad=$ low state energy of the transition, $\mathrm{cm}^{-1}$

$h \quad=$ Planck constant

$C \quad=$ speed of light

$R \quad=$ intensity ratio

$i \quad=$ index for selected transition

\section{Introduction}

$\mathrm{N}$ umerous combustion diagnostic techniques have been developed for measurements of temperature and species concentration. In these methods, laser-based optical techniques offer significant advantages for non-intrusive investigation of combustion process[1]. Laser absorption spectroscopy has been proven valuable as an effective diagnostic technique particularly in applications where the time dependence of the gas flow is of primary interest. Line of Sight absorption can provide both space averaged temperature and concentration information. With the development of laser capability, measurement incorporate with two or more tunable diode lasers for multi parameter resolution is possible, and the technique has been realized by several works[2-6].

\footnotetext{
* Associate professor, Institute of Mechanics, CAS, email: xlyu@imech.ac.cn.

${ }^{\dagger}$ Graduate student, Institute of Mechanics, CAS, email: lifei@imech.ac.cn.

‡ Professor, Institute of Mechanics, CAS, email: lhchen@imech.ac.cn, member AIAA

$\S$ Professor, Institute of Mechanics, CAS, email: xychang@imech.ac.cn, member AIAA.
} 
It is very important to measure temperature and water-vapor concentration for evaluating combustion efficiency in scramjet combustor. Well attention has been paid to the thermometry design based on tunable diode- laser absorption spectroscopy (TDLAS) due to its advantages of high sensitivity, quick response, as well as system simpleness. The use of two tunable diode lasers (TDL) makes it low cost and simple to operate. Temperatures may be inferred from the ratio of the measured line intensity attenuation of individual spectral features.

The aim of this paper is to give an overview of our progress in development of TDLAS thermometry for combustion diagnostics. We mainly concentrate on system construction and calibration of measurement accuracy. Successful experiments were performed in a $\mathrm{CH}_{4} / \mathrm{Air}$ premixed flat flame burner using direct absorption wavelength scanned method with $1 \mathrm{KHz}$ scan rate. Because of the cooler shear layer in the flat flame, mixing layer theory was applied to correct TDLAS results. Comparison with thermocouple measurements, the maxium error of the technique is less than $80 \mathrm{~K}(6.7 \%)$.

\section{Theory}

For a narrow-line light pass through absorbing gas with length $L$, the intensity attenuation of light can be describe by Beer-lambert law:

$$
I_{v}=I_{v, 0} \exp \left(-k_{v} L\right)
$$

Here, $I_{v, 0}$ represents incident intensity, $I_{v}$ represents transmission intensity, $k_{v}$ represents the spectral absorption coefficient at light frequency $v\left(\mathrm{~cm}^{-1}\right)$. The absorption coefficient is related to pressure of absorbing species $P_{a b s}(\mathrm{~atm})$, the line strength of the transition $S_{i}\left(\mathrm{~cm}^{-2} \mathrm{~atm}^{-1}\right)$, and the line shape function $\phi(v)$ (defined as $\left.\int \phi(v) d v=1\right)$.

The spectral absorption coefficient $k_{v}\left(\mathrm{~cm}^{-1}\right)$ can be given below, which is summed over several lines contributes to the absorption in a particular region:

$$
k_{v}=\sum_{i}^{N} P_{a b s} S_{i}(T) \phi_{i}(v)
$$

The line strength is temperature-dependent function, and it may be expressed as:

$$
S_{i}(T)=S_{i}\left(T_{0}\right) \frac{Q\left(T_{0}\right)}{Q(T)}\left(\frac{T_{0}}{T}\right) \exp \left[-\frac{h c E}{k}\left(\frac{1}{T}-\frac{1}{T_{0}}\right)\right]\left[\frac{\left.1-\exp \left(\frac{-h c v_{0}}{k T}\right)\right]}{\left[1-\exp \left(\frac{-h c v_{0}}{k T_{0}}\right)\right]}\right.
$$

where, $Q$ is partition function, $E$ " is low state energy of the transition, $h$ is Planck constant, and $c$ is speed of light.

The intensity ratio $\mathrm{R}$ for two absorption lines can be found by integrating equation (2):

$R=\frac{\int P \cdot X_{H_{2} O} \cdot L \cdot S_{1}(T) \phi_{1}\left(v-v_{01}\right) d v}{\int P \cdot X_{H_{2} O} \cdot L \cdot S_{2}(T) \phi_{2}\left(v-v_{02}\right) d v}=\frac{S_{1}(T)}{S_{2}(T)}=\frac{S_{1}\left(T_{0}\right)}{S_{2}\left(T_{0}\right)} \exp \left[-\frac{h c}{k}\left(E_{1}^{\prime \prime}-E_{2}^{\prime \prime}\right)\left(\frac{1}{T}-\frac{1}{T_{0}}\right)\right]$

For a given pair of lines, the intensity ratio is a function of temperature only, and sensitivity is defined as following:

sensitivit $y=\frac{d R / R}{d T / T}=\frac{h c}{k}\left|\frac{E_{1}^{\prime \prime}-E_{2}^{\prime \prime}}{T}\right|$

The rules of selection of line pair for water vapour thermometry briefly presents here: first, sufficient signal intensity should be detected. From equation (2), absorption coefficient $k_{v}$ is proportional to line strength $S_{i}$. That means, selected lines should have large line strengthen. Otherwise, the sufficient intensity attenuation can not be detected for concentration resolution. Secondly, selected lines cannot be overlapped by neighbouring lines. Possible isolated lines should be selected for avoiding interference with neighboring lines.Thirdly, selected line pair should have enough low state energy difference to fulfil requirement of sensitivity.

Following above rules, spectral calculation in the region of 1300-1500nm is proceeded with HITRAN2004[7], and represents here one pair with wave number $7168.437 \mathrm{~cm}^{-1}$ and $7185.597 \mathrm{~cm}^{-1}$ (both two near $1393 \mathrm{~nm}$ and diode laser is availble elsewhere in commercial market), which can satisfy the requirement for temperature measurement. 
The calculation aims at typical combustion condition with the absorption length about $10 \mathrm{~cm}$ and water vapor concentration about $20 \%$ for typical combustion products (mole fraction ). Fig.1 (a) presents absobance of water vapor absorption in the wavelength range from $7160 \mathrm{~cm}^{-1}$ to $7195 \mathrm{~cm}^{-1}$. Fig.1 (b) and (c) presents indivadual line shapes about selected line pair. The figure shows free of interference with neighbouring absorption lines in concerned temperature range

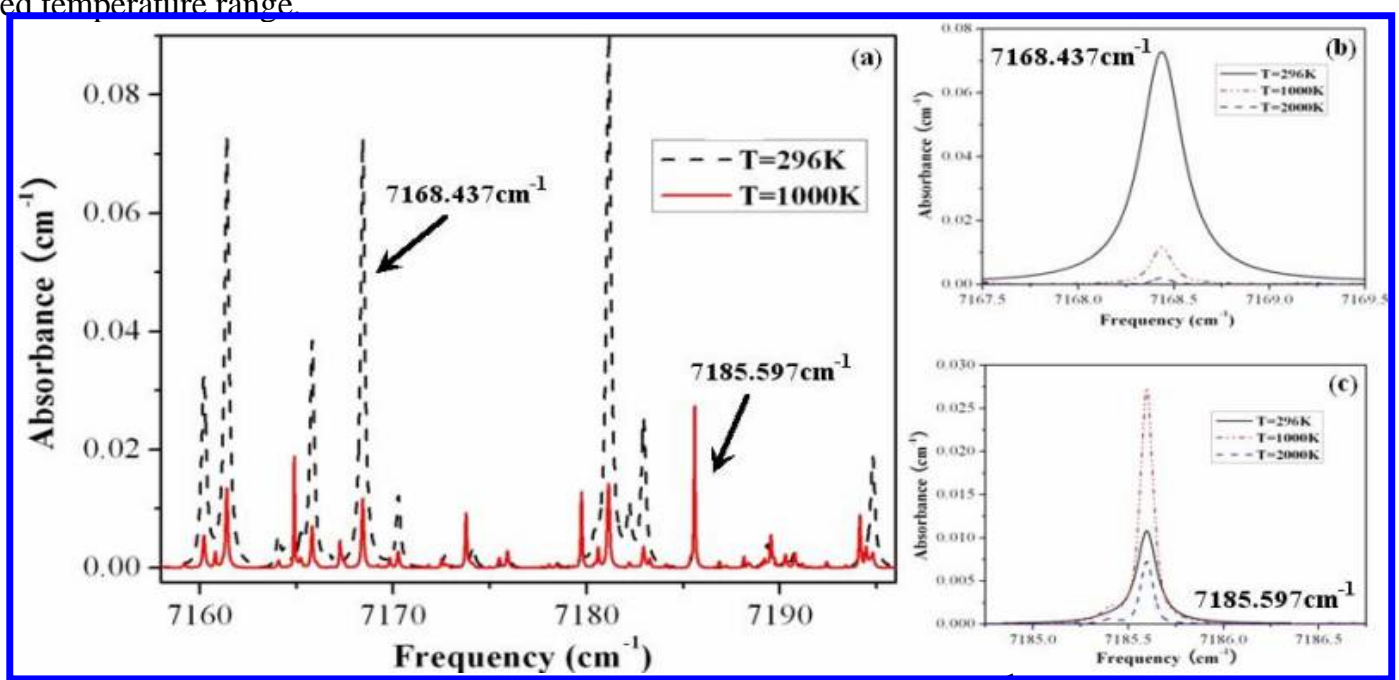

Fig1. HITRAN simulation of water vapor absorption near $7180 \mathrm{~cm}^{-1}$ for different temperature ;(a) wavelength range from $7160 \mathrm{~cm}^{-1}$ to $7195 \mathrm{~cm}^{-1}$;(b) the individual line shape about $7168.437 \mathrm{~cm}^{-1}$;(c) the individual line shape about $7159.597437 \mathrm{~cm}^{-1}$.

Fig.2 illustrates line strengthen of selected line pair versus temperature. The results show $7168.437 \mathrm{~cm}^{-1}$ line has much more strong absorption in low temperature region below $800 \mathrm{~K}$, while $7185.597 \mathrm{~cm}^{-1}$ line has much more strong absorption above the temperature. As a result, the curve for temperature resolution about line pair strengthen ratio is given in Fig 3
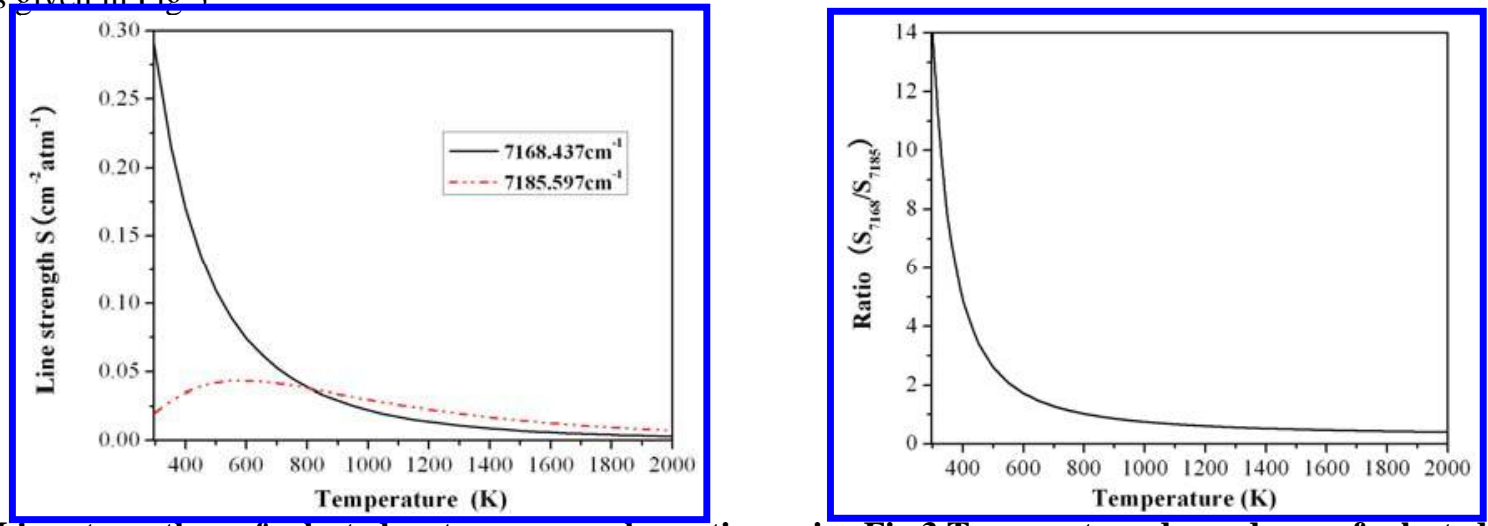

Fig.2 Line strengthen of selected water vapour absorption pair Fig.3 Temperature dependence of selected line nair strenthen ratio

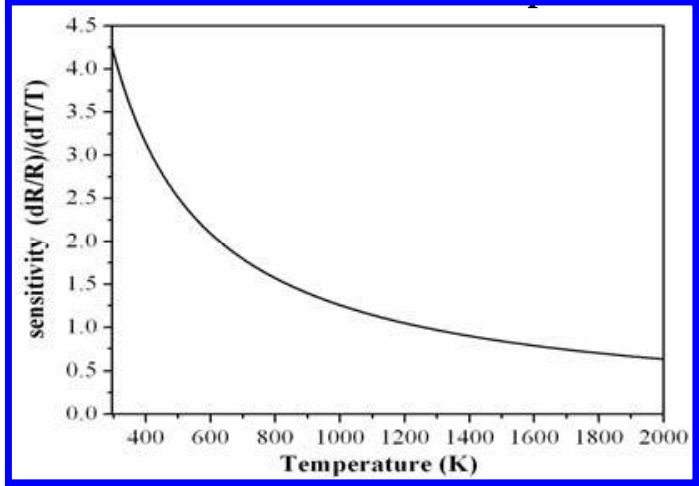

Fig.4 Temperature sensitivity versus temeperature 
The sensitivity is calculated according to Eq.(5) and result indicates that sensor system has the desired sensitivity over the selected temperature range from $400 \mathrm{~K}$ to $2000 \mathrm{~K}$.

\section{Experimental Method}

Figure 5 is schematic diagram of the multiplexed diode-laser sensor system for scanned-wavelength scheme.The system composed of two independently operated DFB diode laser(products of NTT Corporation). The line width of lasing wavelength of both two laser is less than $10 \mathrm{MHz}$. The ramp signal from Signal generator(Model:Tektronix AFG3021) was injected into combination diode/TEC controller (model ITC502, Thorlabs INC.) and lasing wavelength was tuned over the desired transition with the repetition rate of $1 \mathrm{KHz}$.Both the two lasing beam were coupled into one single mode fiber. Then collimated two beam passed through flame generated by a standard premixed $\mathrm{CH}_{4} /$ air flat burner. The intensity was detected by Infrared-sensitive 3-mm diameter InGaAs detector with rising time 30ns. A Tektronix high dynamic oscillationscope(Model: DPO4032, Texktronix INC.) was employed for data record.

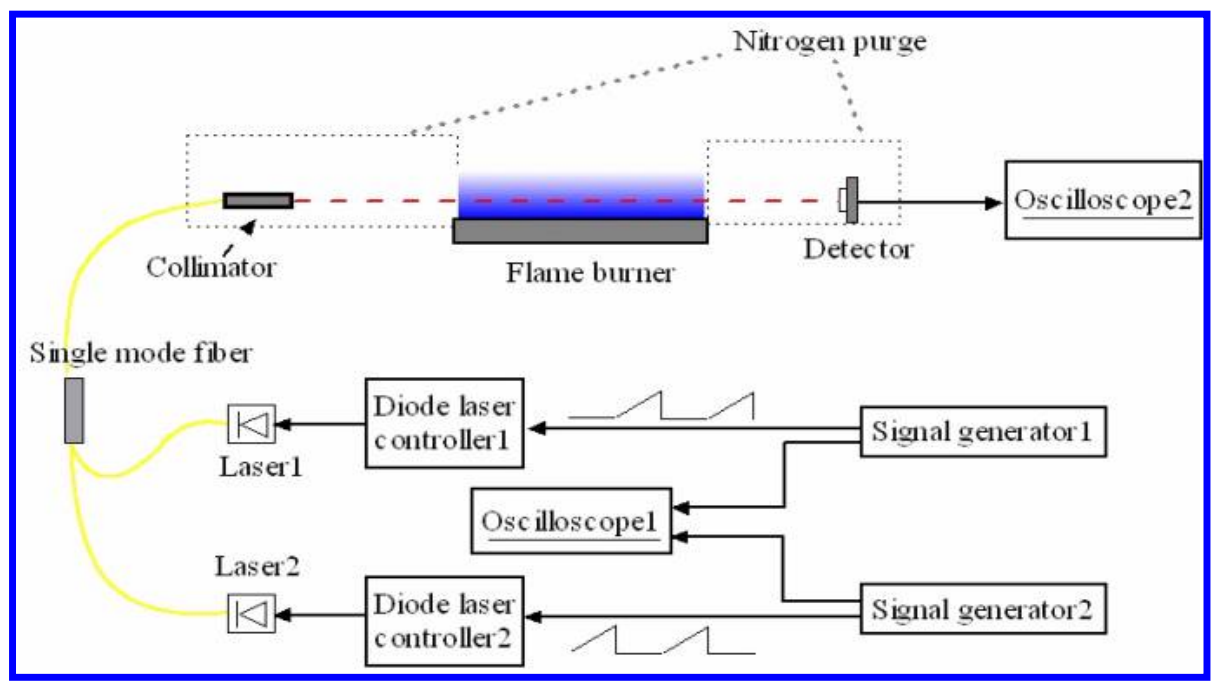

Fig.5 Schematic diagram of TDLAS system

In the present system, only one InGaAs photo-detector was used for transmission intensity record and we employ a scheme called Time-Division-multiplexing(TDM) technique. That means, two signals were alternate in time, and this absorption at two different wavelength can be measured with one detector. Even the technique leads to some uncertainty for the rapid change condition, it is sufficient for current flat burner steady experiment.

For eliminating interference from water vapor contained in ambient atmosphere, dry nitrogen gas was injected slowly into space around burner, collimator and detector during test.

The flat burner is Mckenna-type burner, which flow rate of $\mathrm{CH}_{4}$ and air was controlled through high accuracy flowmeter. The burner is with structure of round porous metal for flame passage at center region and surrounded separate porous metal ring for shroud flow of nitrogen for flame restriction. The diameter of flame region is $60 \mathrm{~mm}$. The B type thermocouple was installed along radius direction for space temperature measurement of the flame. The thermocouple measurement and laser beam path were in same plane.

In previous work, the wavelength was monitored by wavemeter and F-P interferometers. In our experiment, we calibrated the lasing wavelength through known absorption lines, then dependence of wavelength on injection current for certain stabilized temperature was deduced. The relationship was employed for wavelength modulation calibration and line shape monitor, and conversion from time to spectral frequency in the transmission signal was also processed by the method.

\section{Results and Data Analysis}

In the experiment, stoichiometric ratio of $\mathrm{CH}_{4} /$ air mixture was controlled from 0.55 to 1.20 and measured temperature by thermocouple was from $1100 \mathrm{~K}$ to $1500 \mathrm{~K}$. Figure 6 shows transmission signals that were obtained by tuning lasers at $1 \mathrm{KHz}$ rate across $\mathrm{H}_{2} \mathrm{O}$ absorption features near $7168.437 \mathrm{~cm}^{-1}$ and $7185.597 \mathrm{~cm}^{-1}$ by Time Division Multiplexing scheme. The measurement arrangement is in single pass configuration with 60mm-long path. The absorption profiles for line $7168.437 \mathrm{~cm}^{-1}$ and $7185.597 \mathrm{~cm}^{-1}$ were processed by voigt fitting. The signal to noise 
$(\mathrm{S} / \mathrm{N})$ of both two lines were approximately 10 . Ratio of absorbance integration over line shape profile of selected line pair for temperature determination is solved by using equation (4)

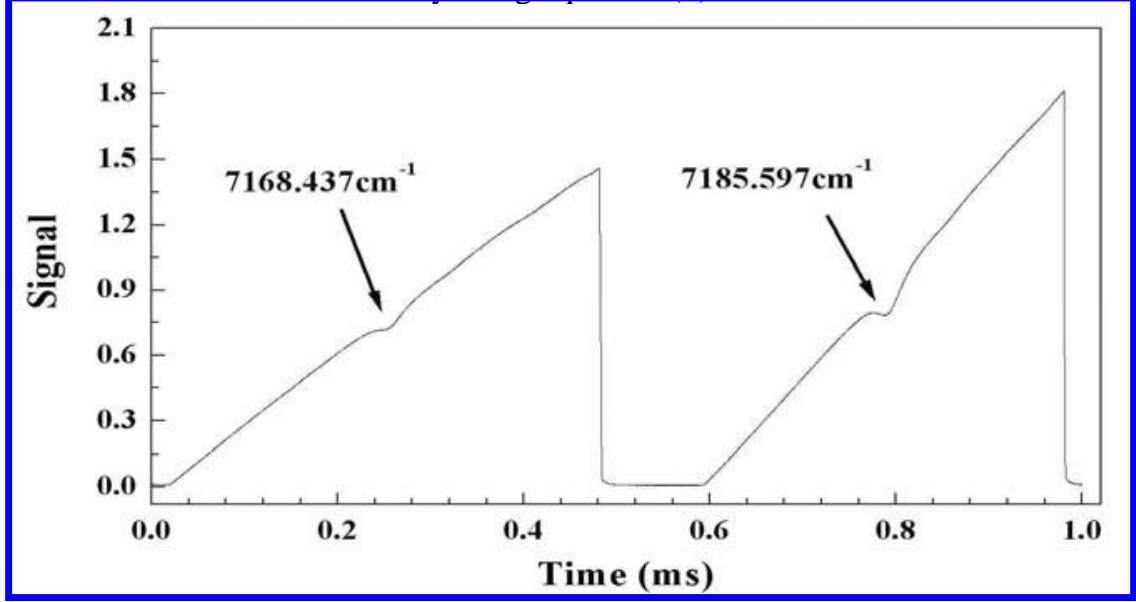

Fig.6 Shematic of Time Division Multiplexing transmission signal

Figure 8 shows comparison between TDLAS thermometry result and thermocouple measurement. It should be noted here that TDLAS thermometry result was corrected with the trapezoid temperature distribution profile of flat flame burner. Compared to thermocouple measurements, the maxium error of the technique is less than $80 \mathrm{~K}(6.7 \%)$.

Figure 8 compares water vapor concentration measurement from TDLAS sensor with chemical equilibrium calculation predicted result at measured temperature. Approximate $10 \%$ uncertainty is ascribed to fluctuation of inlet gas flow rate and uncertainty of line strength at high temperature.

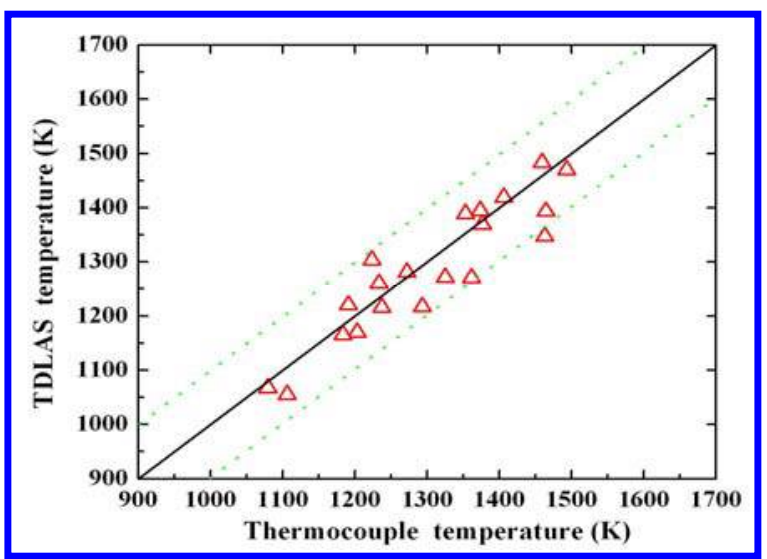

Fig.7 Comparison of TDLAS temperature and thermocouple measurement

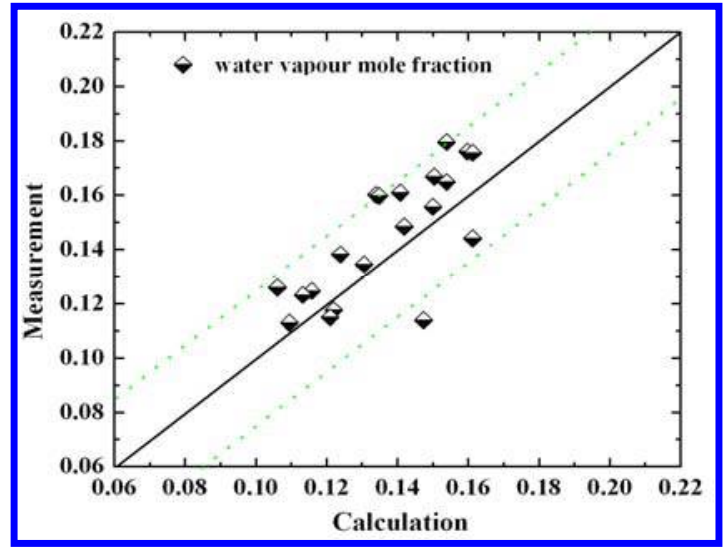

Fig.8 Comparison of water vapor concentration measurement and equilibrium flame prediction.

\section{Conclusions}

This paper is to give an overview of our progress in development of TDLAS thermometry for combustion diagnostics. A multiplexed tunable diode laser system capable of simultaneous measurement of temperature and water vapor concentration has been performed in a $\mathrm{CH}_{4} /$ Air premixed flat flame burner using direct absorption wavelength scanned method with $1 \mathrm{KHz}$ scan rate. Because of the cooler shear layer in the flat flame, mixing layer theory was applied to correct TDLAS results. Comparison with thermocouple measurements, the maxium error of the technique is less than $80 \mathrm{~K}(6.7 \%)$. The system will be developed into multiple species and multiple parameter measurement for real time diagnostics in the combustion chamber. 


\section{References}

${ }^{1}$ N. Docquier, S. Candel, “Combustion control and sensors: a review,” Progress in Energy and Combustion Science, Vol.28, 2002, pp. 107-150

${ }^{2}$ Alan D. Griffiths, A. Frank P. Houwing. “Diode laser absorption spectroscopy of water vapour in a scramjet combustor.”, Applied Optics,Vol.44,2005,pp6653-6659

${ }^{3}$ X. Liu, J.B. Jeffries, R.K. Hanson, K.M. Hinckley, M.A.Woodmansee, "Development of a tunable diode laser sensor for measurements of gas turbine exhaust temperature”, Appl. Phys. B,Vol. 82, 2006,pp469-478

${ }^{4}$ X. Zhou, X. Liu, J.B. Jeffries, R.K. Hanson, “"Development of a Sensor for Temperature and Water Vapor Concentration in Combustion Gases using a Single Tunable Diode Laser.”, Meas. Sci. and Technology,Vol.14, 2003,pp1459-1468

${ }^{5}$ Richter D, Lancaster DG, Tittel FK. "Development of an automated diode-laser-based multicomponent gas sensor”, Appl. Opt.,Vol.39, 2000,pp4444-50.

${ }^{6}$ Teichert H, Fernholz T, Ebert V. "'Simultaneous in situ measurement of CO, H2O and gas temperatures in a full-sized coal-fired power plant by near-infrared diode lasers. ”, Appl. Opt., Vol.42,2003,pp2043-51.

${ }^{7}$ Rothman LS, Jacquemart D, Barbe A, Benner DC, Birk M, Brown LR, et al., "The HITRAN 2004 molecular spectroscopic database.”, JQSRT, Vol.96,2005,pp139-204. 